Www.jmscr.igmpublication.org

Impact Factor (SJIF): 6.379

Index Copernicus Value: 79.54

ISSN (e)-2347-176x ISSN (p) 2455-0450

crossrefDOI: https://dx.doi.org/10.18535/jmscr/v7i1.70

Journal Of Medical Science And Clinical Research

IGM Publication

An Official Publication of IGM Publication

\title{
Clinical investigative profile of Neonatal septicaemia and outcome at tertiary care rural hospital
}

\author{
Author \\ Aatif Sattar \\ MD Paediatrics, JR3 PMT PIMS Loni, India \\ Corresponding Author
}

Aatif Sattar

\begin{abstract}
Background: Neonatal septicaemia remains one of the leading causes of morbidity and mortality both among term and preterm infants. signs and symptoms of neonatal sepsis are nonspecific. ${ }^{[3]}$ These include fever or hypothermia, respiratory distress including cyanosis and apnoea, feeding difficulties, lethargy or irritability, hypotonia, seizures, bulging fontanel, poor perfusion, bleeding problems, abdominal distention, hepatomegaly, gauiac-positive stools, unexplained jaundice Neonatal sepsis is of two types: Early onset Sepsis (EOS): Early onset sepsis presents within first 72 hours of life Late onset Sepsis (LOS): Late onset sepsis commonly presents next 72 hours of age. The cause of infection is either one nosocomial or community acquired and neonates typically existent with septicaemia, pneumonia or meningitis.

Aim: Clinical investigative profile of Neonatal septicaemia and outcome at tertiary care rural hospital.

Objective: to study the various clinical features of neonatal septicaemia for its early identification, to study the result of various investigation in identification of early and late onset neonatal septicaemia to identify various factor leading to neonatal septicaemia.

Material and Methods: A prospective and observational study was carried out Pravara institute of Medical Sciences, which is located at Loni.

Study Population: A total of 130 febrile subjects aged up to 28 days with clinical features such as Babies with birth asphyxia and congenital anomalies were excluded from the study.

Observation and Result: The most common symptoms seen were lethargy and refusal of feeds in $61.66 \%$ and $55.9 \%$ respectively, followed by apnoea and respiratory distress in $34.17 \%$ and $21.67 \%$ respectively. Vancomycin and colistin showed $100 \%$ efficacy against Gram-positive isolates while amikacin, meropenem and linezolid was found to be most effective among Gram-negative isolates in both EOS and LOS.
\end{abstract}

\section{Introduction}

Neonatal septicaemia remains one of the leading causes of mortality and morbidity between preterm and term infants. ${ }^{[1]}$ Even though progresses in neonatal carefulness have enhanced survival and reduced complications in preterm infants, sepsis still contributes significantly to mortality and morbidity among very-low-birthweight (VLBW, <1500 g) infants in Neonatal Intensive Care Units (NICUs). ${ }^{[2]}$ 
The signs and symptoms of neonatal sepsis are nonspecific. ${ }^{[3]}$ These include fever or hypothermia, respiratory distress including cyanosis and apnea, feeding difficulties, lethargy or irritability, hypotonia, seizures, protruding fontanel, bleeding problems, reduced perfusion, hepatomegaly, abdominal distention, unexplained jaundice, gauiac-positive stools, or more importantly, "just not looking right". ${ }^{[4]}$ Infants with hypoxia-acidosis may gasp in utero and lead to pneumonia and meconium aspiration. ${ }^{[5]}$

Definition: National Neonatal Forum of India has defined neonatal sepsis as follows:

Probable (Clinical) Sepsis: In an infant having clinical picture suggestive of septicemia, if there is the presence of any one of the following criteria:

- Existence of predisposing factors: maternal pyrexia or filthy smelling liquor or ruptured of membranes prolonged (>24 hrs) or gastric polymorphs ( $>5$ per high power field).

Positive septic screen - consistent of two of the four parameters namely, TLC $(<5000 / \mathrm{mm})$, band to total polymorphonuclear cells ratio of $>0.2$, absolute neutrophil count $<1800 /$ cumm, Creactive protein $(\mathrm{CRP})>1 \mathrm{mg} / \mathrm{dl}$ and microESR $>$ $10 \mathrm{~mm}$-first hour.

- Radiological evidence of pneumonia. ${ }^{[6]}$

Culture Positive Sepsis: In an infant presence of clinical picture indicative of septicaemia, pneumonia or meningitis, if there is consist of either of the following:

- Separation of pathogens from CSF or abscess (es) or urine or blood

- Pathological evidence of sepsis on autopsy.

\section{Classification}

Neonatal sepsis is of two types:

Early onset Sepsis (EOS): Early onset sepsis presents within first 72 hours of life. In chronic cases the neonate may be symptomatic in utero (poor beat to beat variability, fetal tachycardia).

Clinically, the neonate typically presents as respiratory distress and pneumonia. Occurrence of the subsequent risk factors has been accompanying with an augmented risk of EOS:
- Low birth weight $(<2500 \mathrm{gms})$ or preterm baby

- Febrile illness in the mother within 14 days before delivery

- Foul smelling and/or meconium stained liquoramnii.

- Rupture of membrane prolonged (> 24 hours).

- More than 3 vaginal assessments during labor.

- Prolonged and difficult delivery with instrumentation.

- Perinatal suffocation (Apgar score $<4$ at 1 minute of age) or difficult artificial respiration.

Neonates with presence of filthy smelling liquor or 3 of above stated risk issues should be considered to have EOS \& treated with antibiotics. Presence of $\geq 2$ risk factors should be investigated with sepsis screen and treated accordingly.

Late onset Sepsis (LOS): Late onset sepsis usually presents after 72 hours of age. The source of infection is any of community acquired or nosocomial and neonates usually present with pneumonia, septicaemia, or meningitis.

Risk factors for development of LOS include:

- NICU admission

- Poor hygiene

- Low birth weight (LBW)

- Poor cord care

- Prematurity

- Bottle feeding

- Invasive procedure

- Superficial infection (pyoderma, umbilical sepsis)

- Prelacteal feeding

- Ventilation

- Aspiration of feeds ${ }^{[7]}$

Aim

Clinical investigative profile of Neonatal septicaemia and outcome at tertiary care rural hospital

\section{Objectives}

To study the various clinical features of neonatal septicaemia for its early identification, 
To study the result of various investigation in identification of early and late onset neonatal septicaemia

To identify various factor leading to neonatal septicaemia

\section{Material and Methods}

A prospective and observational study was carried out Pravara institute of Medical Sciences, which is located at Loni.

\section{Study Population}

A total of 130 febrile Subjects aged up to 28 days with clinical features such as Babies with congenital anomalies and birth suffocation were omitted from the study. Informed consent was obtained from parents of the neonates in the study. Detailed maternal, birth, postnatal histories were recorded in a pre-set proforma. The following signs were looked for in the neonates: lethargy, refusal of feeds, respiratory distress $(>60 / \mathrm{min})$, grunt, vomiting, abdominal distension, jaundice, apnoea, seizures, central cyanosis, bradycardia, (HR>160/min), hypothermia (rectal temperature $37.5^{\circ} \mathrm{C}$ ), sclerema. All neonates underwent sepsis screen testing that included total leucocyte count, absolute neutrophil count, Immature to total ratio, serum CRP, micro ESR, blood culture and sensitivity was done in all neonates under aseptic precautions as per standard protocol. The neonates were categorised as (a) proven sepsis- blood culture positive and clinical signs and symptoms of sepsis, (b) Probable sepsis- clinical signs and symptoms of sepsis with two or more parameters of sepsis screen positive and no progress in blood culture. All neonates were stabilised and managed as per standard NICU protocols. Data was analysed using descriptive analytical tools.

\section{Sample Collection}

One millilitre $(\mathrm{mL})$ of blood samples were collected aseptically by trained nurse or lab technician with the help of sterile needle and syringe by venepuncture and instantly the blood samples were cautiously transmitted into $9 \mathrm{~mL}$ of
Brain Heart Infusion (BHI) broth and categorized with the identification number, patient's name, age/sex, date, and time of collection.

\section{Bacteriological Processing}

The BHI broth inoculated with blood sample was transported to the laboratory and incubated at $37^{\circ} \mathrm{C}$ in aerobic condition. Subcultures were prepared in chocolate agar, sheep blood agar, and MacConkey agar after overnight of aerobic incubation. Chocolate agar plates were incubated overnight at $37^{\circ} \mathrm{C}$ in $5 \% \mathrm{CO}_{2}$ atmosphere whereas MacConkey agar and Blood agar plates were incubated overnight at $37^{\circ} \mathrm{C}$ in aerobic atmosphere. Thereafter, culture bottles were observed for turbidity for up to 10 days. Last blind subcultures were prepared in advance reporting the sample negative. Growth found was recognized by standard methods ${ }^{[16]}$. A pureness plate was performed to confirm that the inoculum used for the biochemical tests was pure.

\section{Antibiotic Susceptibility Testing}

All the isolates developed were exposed to antibiotic susceptibility testing by modified KirbyBauer disk diffusion method in compliance with Clinical and Laboratory Standards Institute (CLSI) guidelines using Mueller-Hinton agar standard media. The inhibition zone standards for antimicrobial susceptibility were considered from tables for interpretative zone diameters of CLSI. ${ }^{[8]}$ Antibiotic disks (HiMedia Laboratories, Pvt. Limited, India) used were erythromycin $(15 \mu \mathrm{g})$, oxacillin $(1 \mu \mathrm{g})$, clindamycin $(2 \mu \mathrm{g})$, teicoplanin $(30 \mu \mathrm{g})$, vancomycin $(30 \mu \mathrm{g})$, penicillin $(10 \mathrm{U})$, cotrimoxazole $(25 \mu \mathrm{g})$, cephalexin $(30 \mu \mathrm{g})$, amikacin $(30 \mu \mathrm{g})$, gentamicin $(10 \mu \mathrm{g})$, ofloxacin $(5 \mu \mathrm{g})$, cefotaxime $(30 \mu \mathrm{g})$, cefixime $(5 \mu \mathrm{g})$, ceftazidime $(30 \mu \mathrm{g}), \quad$ piperacillin-tazobactam $(100 / 10 \mu \mathrm{g})$, piperacillin $(100 \mu \mathrm{g}), \quad$ ampicillin $(10 \mu \mathrm{g})$ and carbenicillin $(100 \mu \mathrm{g})$.

Staphylococcus aureus ATCC 25923 and Escherichia coli 25922 were used as a control organisms for antibiotic sensitivity testing. 


\section{Ethical Aspects}

This study was approved by the Institutional Review Committee of Pravara institute of Medical Sciences, which is located at Loni. Informed consent was acquired from the guardians of participating neonates before collecting the specimens.

\section{Observation and Result}

\section{Gender-Wise Distribution of Cases}

Of total 130 enrolled neonates, $68.4 \%$ were males and $31.5 \%$ were females. Among total enrolled cases, among which EOS was found in 89 (68.4\%) neonates while LOS was found in 41 $(31.5 \%)$ neonates (Table 1$)$.

Table 1: Sex-wise distribution of total and septicaemia cases

\begin{tabular}{|l|c|c|c|c|}
\hline \multirow{2}{*}{ Sex } & Total cases (\%) & \multicolumn{3}{|c|}{ Positive cases (\%) } \\
\cline { 3 - 5 } & & Early-onset & Late-onset & Total cases (\%) \\
\hline Male & $89(68.4)$ & $64(69.5)$ & $25(65.7)$ & $89(68.4)$ \\
\hline Female & $41(31.5)$ & $28(30.4)$ & $13(34.2)$ & $41(31.5)$ \\
\hline Total & $130(100)$ & $92(100)$ & $38(100)$ & $130(100)$ \\
\hline
\end{tabular}

Table 2: Clinical features in neonates

\begin{tabular}{|l|c|c|c|}
\hline Clinical features & n (\% of babies) & Proven sepsis $\mathrm{n}(\%)$ & Probable sepsis n (\%) \\
\hline Lethargy & $74(61.66)$ & $40(54.05)$ & $34(45.95)$ \\
\hline Refusal of feeds & $66(55.0)$ & $42(63.64)$ & $24(36.36)$ \\
\hline Apnoea & $41(34.17)$ & $26(63.41)$ & $15(36.59)$ \\
\hline Respiratory distress & $26(21.67)$ & $16(61.54)$ & $10(38.46)$ \\
\hline Jaundice & $22(18.33)$ & $14(63.63)$ & $8(36.36)$ \\
\hline Vomiting & $20(16.67)$ & $8(40.0)$ & $72(60.0)$ \\
\hline Tachycardia & $15(12.5)$ & $8(53.33)$ & $9(46.66)$ \\
\hline Abdominal distension & $14(11.66)$ & $5(35.72)$ & $4(33.33)$ \\
\hline Seizures & $12(10.0)$ & $8(66.67)$ & $4(40.0)$ \\
\hline Grunt & $10(8.33)$ & $6(60.0)$ & $3(30.0)$ \\
\hline Hyperthermia & $10(8.33)$ & $7(70.0)$ & $3(37.5)$ \\
\hline Hypothermia & $8(6.66)$ & $5(62.5)$ & $2(40.0)$ \\
\hline Bradycardia & $5(4.16)$ & $3(60.0)$ & $0(25.0)$ \\
\hline Central cyanosis & $4(3.33)$ & $3(75.0)$ & $0(0)$ \\
\hline Sclerema & $1(0.83)$ & $1(100.0)$ & $(46.6 \%)$ \\
\hline
\end{tabular}

The clinical signs and symptoms seen in the neonates are shown in Table 2. The most common symptoms seen were lethargy and refusal of feeds in $61.66 \%$ and $55.9 \%$ respectively, followed by apnoea and respiratory distress in $34.17 \%$ and $21.67 \%$ respectively. Lethargy, refusal of feeds, apnoea, grunt, respiratory distress and seizures were more common in neonates with proven sepsis than in those with probable sepsis; vomiting and abdominal distension were seen to a greater extent in probable sepsis group than in proven sepsis group.

\section{Isolates Distribution}

Among a total of 130 bacterial isolates recovered, $74(63.8 \%)$ were Gram-positive isolates and 42 (36.2\%) were Gram-negative isolates. Of total positive cases, CoNS were recovered from nearly half of the cases (46.6\%) followed by S. aureus (14.6\%), Acinetobacter spp. and Klebsiella pneumoniae $(7.7 \%)$ whereas viridans streptococci was recovered from a only in 1 case. E. coli and Proteus mirabilis were recovered from LOS cases but not from EOS cases while Enterococcus spp., viridans streptococci, and Burkholderia spp. were recovered from EOS case but not from LOS cases (Table 3). 
Table 3: Distribution of isolated organisms

\begin{tabular}{|l|c|c|c|}
\hline & \multicolumn{3}{|c|}{ Frequency } \\
\hline Organism isolated & Early-onset (EOS) & Late-onset (LOS) & Total (\%) \\
\hline Gram-positive organisms & $\mathbf{6 0}$ & $\mathbf{1 4}$ & $\mathbf{7 4}(\mathbf{5 6 . 9})$ \\
S. aureus & 12 & 5 & $17(13)$ \\
CoNS & 45 & 9 & $54(41.5)$ \\
Enterococcus spp. & 2 & 0 & $2(1.5)$ \\
Viridans streptococci & 1 & 0 & $1(0.76)$ \\
\hline Gram-negative organisms & $\mathbf{3 2}$ & $\mathbf{2 4}$ & $\mathbf{4 6}(\mathbf{3 5 . 3})$ \\
Acinetobacter spp. & 10 & 5 & $15(11.5)$ \\
Pseudomonas aeruginosa & 6 & 2 & $8(6.1)$ \\
Citrobacter spp. & 2 & 2 & $4(3.0)$ \\
E. coli & 1 & 5 & $6(4.6)$ \\
Enterobacter spp. & 2 & 5 & $7(5.3)$ \\
Klebsiellapneumoniae & 9 & 3 & $11(8.4)$ \\
Burkholderia spp. & 2 & 0 & $2(1.5)$ \\
Proteus mirabilis & 0 & 2 & $2(1.5)$ \\
\hline Total & $\mathbf{9 2}$ & $\mathbf{3 8}$ & $\mathbf{1 3 0}(\mathbf{1 0 0})$ \\
\hline
\end{tabular}

\section{Antibiotic Resistance Characteristics of} Isolates Recovered from EOS Septicaemia

Vancomycin and colistin showed $100 \%$ efficacy against Gram-positive isolates. Almost Grampositive isolates were resistant to erythromycin, penicillin, and gentamicin on the other hand amikacin exhibited a favourable efficacy amongst verified antibiotics. Among Gram-negative isolates, approximately all the isolates were resistant to ampicillin and utmost of the isolates were resistant to cefotaxime and cefixime while meropenem, amikacin, and linezolid were initiate to be utmost effective amongst tested antibiotics.

\section{Antibiotic Resistance Characteristics of Isolates Recovered from LOS}

Of isolates recovered from LOS cases, most of the Gram-positive isolates were resistant to erythromycin, penicillin, and cephalexin while vancomycin and colistin showed $100 \%$ efficacy and amikacin showed better efficacy among all the antibiotics tested. Among Gram-negative isolates, closely all the isolates remained resistant to ampicillin whereas nearly all Citrobacter spp. and Proteus mirabilis were prone to almost of the tested antibiotic. Approximately all isolates of Enterobacter spp., $33.3 \%$ of the Klebsiella spp, and up to $50 \% \mathrm{E}$. coli isolates were resistant to most of the antibiotic tested.

\section{Discussion}

Neonatal sepsis remains a dreaded cause of neonatal mortality and morbidity. The blood culture positivity in LOS in the present study was $42.5 \%$, while $57.5 \%$ had probable sepsis. Roy et al had a blood culture positivity of $47.5 \%$ in their study. ${ }^{[9]}$ In other Indian studies, the blood culture yield has ranged from as low as $25 \%$ to as high as $64.87 \%$ in neonates with sepsis. ${ }^{[10]}$ Among hospitalised neonates, an incidence of LOS varying between $0.4 \%$ to $14.2 \%$ has been reported. ${ }^{[11]}$ Tallur et al in their study of neonatal sepsis reported that $16.5 \%$ had late onset sepsis. [12]

In the present study, lethargy, refusal of feeds and apnoea were the predominant symptoms noted among neonates with LOS. Kar SS et al in their study in 2013 found apnoea as the most common followed by lethargy and tachycardia in neonates with LOS. ${ }^{[13]}$ Cardiorespiratory signs and jaundice were the most frequent clinical features reported by Tallur SS et al. ${ }^{[12]}$ The signs and symptoms of sepsis are non-specific and demand a high degree of suspicion for early diagnosis. ${ }^{[14]}$

The commonest organism causing LOS in the present study was Klebsiella followed by Staphylococcus aureus and coagulase negative Staphylococcus. Waters et al in their review of the etiology of community acquired neonatal sepsis in low and middle income countries found Klebsiella to be highly prevalent in South-East Asia. In 
developing countries, they found potential similarities in major causative organisms between hospital-acquired and community acquired neonatal sepsis. ${ }^{[15]}$ Tallur et al reported also reported Klebsiella species as the most common organism in their study. ${ }^{[12]}$ Vishwanathan R et al in their study in a rural NICU set up, reported $46.3 \%$ blood culture positivity with predominant gram negative isolates, Klebsiella being the most communal microorganism followed by $\mathrm{E}$ coli. They also noted that profile of organisms causing early and late onset sepsis was similar in their study. ${ }^{[16]}$

In 2012, Hammoud MS et al in Kuwait reported CONS as the most common causative organism in $35.7 \%$ of LOS; Klebsiella was the most common gram negative organism in $18.8 \%$ of LOS. ${ }^{[17]}$ Tsai $\mathrm{MH}$ et al reported that rates of LOS were in reverse to gestational age and birth weight. Increased risk of mortality and morbidity was associated with Pseudomonas and Candida SPP in LOS. ${ }^{[18]}$ CONS account for $35.5 \%-47.4 \%$ of LOS in some developing nations and a higher percentage in industrial countries. CONS are emerging as the most common causative organism in LOS. As the pattern of isolates in LOS changes over time and regions, this should be regularly reevaluated to guide management. ${ }^{[19]}$

\section{Conclusion}

The clinical features of neonatal sepsis being nonspecific, pose a great challenge for prompt diagnosis. Lethargy, refusal of feeds and apnoea were the most common clinical features in this study. Klebsiella was predominant gram negative organism and Staphylococcus aureus and CONS were the predominant gram positive isolates from blood culture in EOS and LOS.

\section{Reference}

1. Neonatal Group of Chinese Medical Science Branch, "Editorial board of Chinese Journal of Paediatrics, Chinese Medical Association. Neonatal sepsis diagnosis and treatment program," Chin J Pediatr, vol. 41, pp. 897-899, 2003.

2. E. J. Weston, T. Pondo, M. M. Lewis et al., "The burden of invasive early-onset neonatal sepsis in the united states, 20052008," Paediatric Infectious Disease Journal, vol. 30, no. 11, pp.937-941, 2011.

3. A. Chiabi, M. Djoupomb, E. Mah et al., "The clinical and bacteriogical spectrum of neonatal sepsis in a tertiary hospital in Yaounde, Cameroon," Iranian Journal of Paediatrics, vol. 21, no.4, pp. 441-448, 2011.

4. Y. Wang and S. Qian, "Relevant explanation of the expert consensus for neonatal sepsis shock (infectious shock," Chin J Pediatr, vol. 53, pp. 584-585, 2015.

5. Y. Wen, B. Zhu, A. Huang, and Y. Jiang, "Diagnostic value of combined detection of serum PCT and CRP in patients with sepsis," Exp Lab Med, vol. 34, pp. 231233, 2016.

6. H. Fan, X. Han, and X. Yin, "Research progress of neonatal sepsis," Chin Paediatric Emerg Med, vol. 21, pp. 539542, 2014.

7. D. C. Angus, W. T. Linde-Zwirble, J. Lidicker, G. Clermont, J. Carcillo, and M. R. Pinsky, "Epidemiology of severe sepsis in the United States: analysis of incidence, outcome, and associated costs of care," Critical Care Medicine, vol. 29, no. 7, pp. 1303-1310, 2001.

8. G. S. Martin, D. M. Mannino, and M. Moss, "The effect of age on the development and outcome of adult sepsis," Critical Care Medicine, vol. 34, no. 1, pp. 15-21, 2006.

9. B. Goldstein, B. Giroir, and A. Randolph, "International paediatric sepsis consensus conference: definitions for sepsis and organ dysfunction in paediatrics," Paediatric Critical Care Medicine, vol. 6, no. 1, pp. 2-8, 2005. 
10. Y. Jiang, "Progress in diagnosis and treatment of neonatal septicaemia," Chin J Neonatal, vol. 25, p. 60, 2010.

11. P. R. A. Carvalho, L. Feldens, E. E. Seitz, T. S. Rocha, M. A.Soledade, and E. A. Trotta, "Prevalence of systemic inflammatory syndromes at a tertiary paediatric intensive care unit," Jornal de Paediatric, vol. 81, no. 2, pp. 143-148, 2005.

12. D. Drassinower, A. M. Friedman, S. G. Obi `can, H. Levin, and C. GyamfiBannerman, "Prolonged latency of preterm premature rupture of membranes and risk of neonatal sepsis the abstract was presented at the 35th annual meeting of the Society for Maternal-Fetal Medicine, San Diego, CA, Feb. 2-7,2015.," American Journal of Obstetrics and Gynaecology, vol. 214,no. 6, pp. 743-743.e6, 2016.

13. S. Srinivasa and D. Arunkumar, "Bacterial isolates and their Antibiotic susceptibility patterns in Neonatal sepsis," Current Paediatric Research, vol. 18, no. 2, pp. 83-86, 2014.

14. M. Behnes, T. Bertsch, D. Lepiorz et al., "Diagnostic and prognostic utility of soluble CD 14 subtype (presepsin) for severe sepsis and septic shock during the first week of intensive care treatment," Critical Care, vol. 18, no. 5, article no. 507, 2014.

15. B. Liu, Q. Yin, Y.-X. Chen, Y.-Z. Zhao, and C.-S. Li, "Role of Preseps in (sCD14ST) and the CURB65 scoring system inpedicting severity and outcome of community-acquired pneumonia in an emergency department," Respiratory Medicine, vol.108, no. 8, pp. 1204-1213, 2014.

16. J. L. Leante-Castellanos, L. G. De Guadiana-Romualdo, C.FuentesGuti`errez, A.Hernando-Holgado, A. Garc'ra-Gonz'alez, and E. Jim'enezSantos, "The value of lipopolysaccharide binding protein for diagnosis of late-onset neonatal sepsis in very low birth weight infants," Journal of Perinatal Medicine, vol. 43,no. 2, pp. 253-257, 2015.

17. Y.Wu, "Distribution and drug resistance of pathogenic bacteria in blood culture of children," Chin J Nosocomiology, vol. 16, pp.463-465, 2006.

18. X. Chen and C. Jiang, "Research progress on diagnostic index of bacterial SIRS in new born," Int J Pediatr, vol. 40, pp. 148151,2013.

19. M. Li, L. Dong, and X. Cui, "The 13-year changes of blood cultures in children with septicaemia and the results of drug sensitivity test," J Clin Pediatr, vol. 21, pp. 85-90, 2003. 